\title{
Versión Argentina de la Escala de Gratitud de Alarcón
}

\author{
Alarcon's Argentine Gratitude Scale
}

\section{(1) PSicOGENTE}

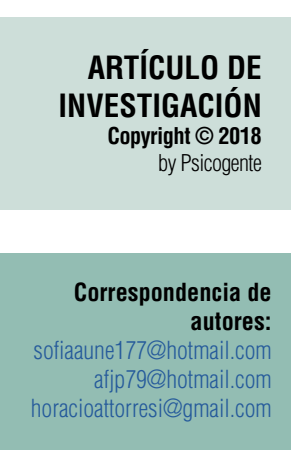

Recibido: 08-11-17 Aceptado: 03-09-18 Publicado: 08-01-19
Resumen

Objetivo: La investigación tuvo como fin la adaptación para adultos argentinos de la Escala de Gratitud de Alarcón (EG)

Método: La EG se administró a 923 participantes (65\% mujeres) cuya media de edad fue de 31 años. Se separó la muestra en dos partes en forma aleatoria. Con una de las submuestras se realizó un Análisis Factorial Exploratorio (AFE) mientras que sobre la otra submuestra se corroboró la estructura hallada en el AFE mediante un Análisis Factorial Confirmatorio (AFC). El AFE se efectuó mediante el método de mínimos cuadrados no ponderados robustos para la extracción de los factores con base en la matriz de correlaciones policóricas y la implementación óptima del análisis paralelo para la determinación del número de dimensiones. El AFC también se basó en la matriz de correlaciones policóricas, estimándose los parámetros con el método de mínimos cuadrados ponderados robustos.

Resultados: Mientras que la estructura factorial de EG era tridimensional, los puntajes de la versión argentina mostraron evidencias de unidimensionalidad. Un modelo monofactorial de 12 ítems describió el $51,28 \%$ de la varianza. El alfa ordinal fue de 0,91 . Se exploraron relaciones entre el puntaje en gratitud y las variables sociodemográficas género, edad, zona de residencia y nivel máximo de educación alcanzado. Se obtuvieron evidencias de validez convergente con la Versión Argentina de la Escala de Eratitud de Lima de Alarcón y la Escala de Conducta Prosocial de Auné, Abal y Attorresi.

Conclusiones: Se concluye que las propiedades psicométricas evidenciadas en el AFE y en el AFC así como el resultado del análisis de la validez convergente de la versión argentina de la EG resultan adecuados. Dada la relación negativa de la gratitud con el neuroticismo, la depresión y la tasa de suicidio, esta escala puede ser utilizada en intervenciones de corte clínico y psicosocial. Al ser unidimensional, el puntaje total de la escala es el puntaje en gratitud, siendo así muy sencilla para administrar y puntuar.

Palabras clave: Medición de la gratitud, Adaptación de Escala de Gratitud de Alarcón, Relación entre gratitud, felicidad y conducta prosocial

\section{Abstract}

Objective: In this paper the Alarcon's Gratitude Scale (GS) adapted for Argentinian adults is showed.

Method: The GS was administered to 923 participants that were sampled as follows: 65\% were women with an average age of 31. The sample was randomly divided in two groups. An Exploratory method of Factor Analysis (EFA) was conducted using one of the sub-samples, at the same time, through a Confirmatory method of Factor Analysis (CFA) the other group's structure results from EFA were corroborated. Also, in order to get factors, based on the polychoric correlation matrix and the optimal implementation of parallel analysis, for determining the number of dimensions, weighted least squares method (EFA) were used; taking into account that parameters were estimated through weighted least squares method. The EFA was based on the polychoric correlation matrix.

Results: While the factorial structure of the GS was three-dimensional, the scores got from Argentine version showed evidences of uni-dimensionality. A 12 items single factor model showed $51,28 \%$ of variance and ordinal alpha was 0,91 . Relationships between the gratitude score and socio-demographic variables such as gender, age, place of residence and maximum level of education reached were explored, showing as

Cómo citar este artículo (APA): Auné, S.E., Abal, F.J.P. y Attorresi, H.F. (2019). Versión Argentina de la Escala de Gratitud de Alarcón. Psicogente 22(41), 1-21. https://doi.org/10.17081/ psico.22.41.3309 
results evidence of convergent validity between the Argentine version of Alarcon's Gratitude Scale of Lima and Auné, Abal and Attorresi's Social Behavior Scale.

Conclusions: It is concluded that the psychometric properties evidenced in the EFA and in the CFA as well as the result of the analysis of the convergent validity of the Argentine version of the GS are adequate. Given the negative relationship of gratitude with neuroticism, depression and suicide rate, this scale can be used in clinical and psychosocial interventions. Being one-dimensional, the total score of the scale is the score in gratitude, thus being very easy to administer and score.

Keywords: Measurement of gratitude, Adaptation of the Alarcón Gratitude Scale, Relationship between gratitude, happiness and prosocial behavior

\section{INTRODUCCIÓN}

La gratitud es un constructo relativamente poco estudiado y solo en los últimos años se ha alcanzado cierto desarrollo y sistematicidad (Wood, Froh, \& Geraghty, 2010). Watkins, Woodward, Stone y Kolts (2003) la consideran uno de los fenómenos abandonados por la Psicología, sobre todo teniendo en cuenta que sus equivalentes lingüísticos existen en prácticamente todos los idiomas y que la mayoría de las religiones en el mundo han destacado su importancia.

McCullough, Emmons y Tsang (2002) definieron la disposición a la gratitud como una tendencia generalizada a reconocer y responder con emoción de agradecimiento a la benevolencia de otra persona. Emmons y McCullough (2003) identificaron conceptualizaciones más amplias en la bibliografía específica, en las cuales se caracteriza a la gratitud como una emoción, actitud, virtud moral, rasgo de personalidad o respuesta de afrontamiento. Asimismo, destacaron que la gratitud posee componentes tanto cognitivos como emocionales.

Watkins et al. (2003) definieron el afecto de gratitud como una apreciación agradecida por los favores recibidos. Identificaron cuatro características de las personas agradecidas. Los individuos agradecidos tienen una sensación de plenitud, valoran las contribuciones de otros para su bienestar, aprecian los placeres simples de la vida y, por último, reconocen la importancia de experimentar y expresar la gratitud.

Según Wood et al. (2010) a un nivel disposicional, la gratitud es parte de una orientación vital más amplia que permite apreciar y tomar conciencia de lo positivo del mundo. Esta orientación vital debe distinguirse de otras emociones tales como optimismo y esperanza, las cuales se centran en el futuro. Por el contrario, la gratitud se focaliza en el presente (Hammer \& Brenner, 2017). 
Distintos estudios han relacionado a la gratitud con cada uno de los Cinco Factores de la Personalidad (Five Factor Model; Digman, 1990), hallando que las personas agradecidas son más extrovertidas, agradables, abiertas, conscientes y más emocionalmente estables (e.g. Wood, Maltby, Gillett, Linley \& Joseph, 2008). En general, se ha encontrado que las personas agradecidas son menos irascibles, hostiles, depresivas y emocionalmente vulnerables, experimentando emociones positivas más frecuentemente (Auné, Abal \& Attorresi, 2016; Wood, et al., 2010).

La gratitud ha correlacionado positivamente con rasgos asociados al funcionamiento social positivo: calidez emocional, sociabilidad, proactividad y altruismo (Auné, Blum, Abal, Lozzia \& Attorresi, 2014). Las personas agradecidas tienen una mayor apertura hacia sus sentimientos, ideas y valores, así como sentido del deber y esfuerzo orientado a logros (Joseph \& Wood, 2010; Wood, et al., 2010).

La gratitud es un predictor robusto del bienestar general (Van Dusen, Tiamiyu, Kashdan \& Elhai, 2015). Es así que la más frecuente asociación de la gratitud en la investigación empírica es con el bienestar subjetivo e incluso lo predice con una considerable precisión (Gulliford, Morgan \& Kristjánsson, 2013). Entre las posibles explicaciones una es que la gratitud es contraria a la envidia y los deseos materialistas y estos últimos, a su vez, disminuyen el bienestar personal. Las personas agradecidas reportaron estar más dispuestas a compartir sus posesiones, ser más generosas con los otros y menos envidiosas del éxito ajeno. Asimismo, manifestaron estar menos de acuerdo con las ideas de que el éxito material está relacionado con la satisfacción vital y de que el bienestar económico conduce necesariamente a la felicidad (McCullough et al., 2002). Otra explicación es hallada en un estudio realizado en 2013, por Kleiman, Adams, Kashdan y Riskind donde encontraron que la gratitud y la perseverancia son factores resilientes que interactúan sinérgicamente potenciando el sentido de la vida. Los individuos con altos niveles de gratitud y perseverancia mostraron significativamente menores tasas de intentos de suicidio que aquellos con cualquier otra configuración de gratitud y perseverancia (Kleiman et al., 2013). De ambos rasgos, la gratitud tuvo mayor efecto, coincidiendo con estudios que ya habían detectado la relación inversa entre gratitud e intentos de suicidio (e.g. Li, Zhang, Li, Li, \& Ye, 2012).

La gratitud podría incluso ayudar a reinterpretar eventos desagradables y mejorar la experiencia de los positivos, crear relaciones personales más fuertes y un crecimiento de los vínculos sociales (Bartlett, Condon, Cruz, 
Baumann \& Desteno, 2012; Emmons \& Mishra, 2011). Los individuos con una mayor gratitud desarrollan significativamente menos síntomas depresivos a lo largo del tiempo (e.g. Disabato, Kashdan, Short, \& Jarden, 2016; Lambert, Fincham, \& Stillman, 2012) así como también presentan una menor tasa de Trastorno de Estrés Postraumático (TEP). La vinculación con el TEP la obtuvieron Israel-Cohen, Uzefovsky, Kashy-Rosenbaum y Kaplan (2015) a partir de una muestra de adolescentes con bajo a medio nivel socioeconómico de una escuela israelí ubicada en una localidad que sufrió ataque de misiles dos meses y medio antes del estudio. Asimismo, Van Dusen et al. (2015) estudiaron la relación entre gratitud y TEP. Para ello, conformaron una muestra de estudiantes universitarios estadounidenses que alguna vez estuvieron expuestos a un evento traumático, encontrando una relación inversa entre gratitud y TEP. La relación negativa más intensa fue con los aspectos del TEP vinculados a alteraciones negativas en el humor y la cognición.

Con respecto a la estructura interna de la gratitud, Hammer y Brenner (2017) señalan que existe un extenso debate y reconocen que actualmente continúa abierto. Los autores puntualizan tres corrientes principales que postulan que: a) la gratitud es un constructo unitario, b) existen formas independientes pero relacionadas de gratitud (modelo de factores correlacionados), o c) la gratitud existe en formas específicas, pero a su vez es un factor independiente general. Esta última postura tiene dos variantes (Hammer \& Brenner, 2017), según las cuales la gratitud es: 1) Un factor general que existe independientemente de sus formas específicas (operacionalizado, por ejemplo, por un modelo bifactorial) o 2) Un factor jerárquico que es definido por el elemento común entre las formas específicas (por ejemplo, operacionalizado mediante un modelo de segundo orden).

Las estructuras postuladas encuentran evidencia en instrumentos psicométricos construidos para medir la gratitud. Entre las medidas más relevantes para medir la gratitud se encuentran las siguientes, ordenadas según el año de publicación:

\subsection{Cuestionario de Gratitud (Gratitude Questionnaire-6 [GQ-6] de}

McCullough et al., 2002)

Es una escala de seis ítems con formato Likert para adultos. Las opciones de respuesta se ordenan desde 1 (Fuertemente en desacuerdo) hasta 7 (Fuertemente de acuerdo). Los ítems miden la frecuencia e intensidad de los sentimientos de gratitud y el rango de eventos o personas objeto de 
los mismos. La estructura unifactorial ha mostrado un adecuado ajuste y la consistencia interna fue muy buena según el criterio de DeVellis (2012) ( $\alpha$ $=0,82$ ).

\subsection{Test de Gratitud, Resentimiento y Apreciación (Gratitude Resentment} and Appreciation Test [GRAT] de Watkins et al., 2003)

Posee tres subescalas que miden: a) Sensación de Plenitud, b) Apreciación de los Placeres Simples y c) Apreciación Social. Son en total 44 ítems que se valoran en una escala Likert de cinco opciones y muestran una consistencia interna excepcional $(\alpha=0,92)$ según el criterio de DeVellis (2012). El test tiene una versión corta revisada (Thomas \& Watkins, 2003). Las muestras utilizadas estuvieron conformadas por estudiantes universitarios.

\subsection{Escala de Apreciación (Appreciation Scale [AS] de Adler \& Fagley, 2005)}

Posee una subescala de Gratitud, formada por diez ítems. La muestra utilizada estuvo conformada por estudiantes universitarios. En cuanto a sus propiedades psicométricas, tuvo una consistencia interna aceptable según el criterio de DeVellis (2012) $(\alpha=0,76)$ y se obtuvieron evidencias de validez convergente con Satisfacción Vital (medido con la Escala de Satisfacción Vital, Satisfaction with Life Scale [SWLS] de Diener, Emmons, Larsen, \& Griffin, 1985) y Afecto Positivo (medido con la Escala de Afecto Positivo y Negativo, Positive and Negative Affect Scale [PANAS] de Watson, Clark, \& Tellegen, 1988) y de validez divergente con Afecto Negativo (medido con PANAS).

\subsection{Cuestionario de Gratitud - 20 ítems ([G-20] de Bernabé-Valero, García-} Alandete \& Gallego-Pérez, 2014)

Los ítems se responden mediante una escala de tipo Likert de siete opciones que indica el grado de acuerdo con una determinada afirmación. La estructura interna es tetrafactorial, siendo las dimensiones: Gratitud Interpersonal ( $\alpha$ $=0,84)$, Gratitud ante el Sufrimiento $(\alpha=0,78)$, Reconocimiento de los Dones $(\alpha=0,75)$ y Expresión de la Gratitud $(\alpha=0,75)$. Los autores utilizaron para su construcción una muestra de estudiantes de distintas especialidades de la carrera de Magisterio de una universidad privada de la Comunidad Valenciana (España).

\subsection{Escala de Gratitud de Alarcón (2014)}

Se encuentra entre los desarrollos más recientes para medir la gratitud. Se basa en una definición operacional del constructo realizada por el autor, el cual lo entiende como una experiencia afectiva que permite al sujeto valorar 
las cualidades positivas de una acción humana. Incluye un deseo de dar las gracias a quien realizó la acción, sentimiento que perdura en el tiempo.

Este trabajo tiene como objetivo adaptar la Escala de Gratitud de Alarcón (2014) a población adulta argentina, aportando evidencias de validez y estudios de confiabilidad. Esta escala es la más próxima culturalmente a Argentina, por tratarse de otro país latinoamericano. El interés en adaptar dicha escala se fundamenta en la escasez de instrumentos construidos o adaptados para el contexto local que permitan medir el constructo.

\section{MÉTODO}

\subsection{Diseño}

Se trata de un tipo de investigación no-experimental dada la ausencia de manipulación de variables independientes y de aleatorización (Montero \& León, 2007) y específicamente de un estudio psicométrico (Ato, López, \& Benavente, 2013).

\subsection{Participantes}

El método de muestreo fue no probabilístico por accesibilidad (Gil-Escudero \& Martínez-Arias, 2001). Se recabaron datos pertenecientes a 923 sujetos adultos, de los cuales 57\% fueron mujeres. La media de edad fue de 31 años ( $D E=11$ años). En cuanto al nivel educativo, el 14\% contaba como máximo con un nivel de secundario incompleto y el $86 \%$ había completado, como mínimo, el nivel secundario. En referencia a la zona de residencia, $20 \%$ provenía de la ciudad de Buenos Aires, $28 \%$ del Gran Buenos Aires, $18 \%$ del resto de la provincia de Buenos Aires y $34 \%$ del resto de las provincias argentinas.

\subsection{Instrumentos}

\subsubsection{Cuestionario Sociodemográfico}

Cuestionario diseñado ad hoc para medir características sociodemográficas de los participantes: género, edad, máximo nivel de educación alcanzado y zona de residencia dentro de Argentina.

\subsubsection{Escala de Gratitud de Alarcón (EG, 2014)}

Sobre la que se realizó la adaptación del instrumento. La Escala de Gratitud de Alarcón (2014) está compuesta por 18 ítems que se responden en una escala Likert de cinco opciones (Totalmente de acuerdo, Acuerdo, Ni acuerdo ni desacuerdo, Desacuerdo y Totalmente en desacuerdo). En el desarrollo 
original de la EG, por medio de un Análisis de Componentes Principales y rotación Varimax se extrajeron tres factores, que, en conjunto, explicaron el $55 \%$ de la varianza. Se indica asimismo el valor del Alfa de Cronbach $(\alpha)$ de la EG original para cada factor:

Reciprocidad (nueve ítems, $\alpha=0,86$ ). Es la satisfacción de corresponder a la persona de quien se recibió un beneficio o favor.

Obligación Moral (siete ítems, $\alpha=0,82$ ). Es el componente moral de la gratitud, el deber de agradecer.

Cualidad Sentimental (dos ítems, $\alpha=0,71$ ). Es el sentimiento positivo, la experiencia de agrado hacia el benefactor.

La muestra utilizada por Alarcón estaba compuesta por 675 participantes peruanos (56\% mujeres) cuya media de edad era 32,50 años. La mayoría eran estudiantes universitarios de instituciones públicas y privadas y graduados.

\subsubsection{Versión Argentina de la Escala de Felicidad de Lima (VAEFL, Auné,} Abal, \& Attorresi, 2017)

Se trata de la adaptación argentina de la Escala de Felicidad de Lima (Alarcón, 2006). Esta versión adaptada se compone de 12 ítems distribuidos en tres dimensiones que explicaron el $69 \%$ de la varianza: Sentido de la Vida Perspectiva Externa (SVPE, cuatro ítems, $\alpha=0,84$, $\alpha$ en este estudio $=0,81$ ), Sentido de la Vida Perspectiva Interna (SVPI, cinco ítems, $\alpha=0,90$, $\alpha$ en este estudio $=0,79$ ) y Satisfacción con la Vida (SCV, tres ítems, $\alpha=0,75$, $\alpha$ en este estudio $=0,76)$. Los ítems se responden según una escala de tipo Likert de cinco alternativas: Totalmente de acuerdo, Acuerdo, Ni acuerdo ni desacuerdo, Desacuerdo y Totalmente en desacuerdo.

\subsubsection{Escala de Conducta Prosocial (ECP, Auné, Abal \& Attorresi, 2016;}

Auné \& Attorresi, 2017)

Este instrumento de 15 ítems modeliza a la conducta prosocial en adultos en dos dimensiones: Ayuda ( $\mathrm{AY}$, ocho ítems, $\alpha=0,85, \alpha$ en este estudio $=0,84$ ) y Confortar ( $\mathrm{CO}$, siete ítems, $\alpha=0,77$, $\alpha$ en este estudio $=0,78)$. Los ítems se responden en una escala Likert de seis opciones que expresan la frecuencia con la que se realiza la acción prosocial ( $1=$ Nunca, $2=$ Casi Nunca, $3=A$ Veces, 4 = Con Frecuencia, 5 = Casi Siempre, 6 = Siempre). 


\subsection{Procedimiento}

En primer lugar, cinco jueces expertos en Psicometría y Psicología Social corroboraron la equivalencia cultural de los reactivos, valorándola en una escala de uno a cinco. Ulteriormente se verificó la claridad y fácil comprensión de los ítems en un ensayo piloto con 35 participantes. Como resultado de esta exploración, se concluyó que no era necesaria la modificación de la redacción original del instrumento.

Posteriormente, se administró consentida, voluntaria y anónimamente la Escala de Gratitud de Alarcón (2014) a la muestra definitiva. Los datos fueron recopilados en forma virtual mediante la plataforma Google Forms sin ofrecer recompensa alguna por la participación.

\subsection{Análisis de datos}

Sobre las respuestas de los jueces expertos, se calculó el índice de concordancia $W$ de Kendall, el cual indicó una alta coherencia en las evaluaciones realizadas. Para comenzar el análisis de los datos resultado de la administración se evaluó el porcentaje de valores perdidos, el cual resultó menor al $5 \%$ de las respuestas en todos los ítems. Estos valores perdidos fueron reemplazados por la media que indicó el ítem en la muestra. Se detectaron datos anómalos multivariados calculando la distancia de Mahalanobis considerando un punto de corte de $p \leq 0,001$ (Tabachnick \& Fidell, 2001). Este análisis señaló a 59 patrones de respuesta como anómalos, y consecuentemente los protocolos correspondientes fueron eliminados de la base de datos definitiva. Por lo tanto, el nuevo tamaño muestral fue de 864 participantes.

Posteriormente, se exploró la normalidad multivariante de los datos depurados mediante el índice de Mardia (1970) -el cual evalúa asimetría y curtosis multivariante-, calculado con el macro para SPSS desarrollado por DeCarlo (1997). Además, se obtuvieron estadísticos descriptivos de las respuestas a cada ítem: media (M), mediana (Mdn), desvío estándar (DE), asimetría univariante (As) y curtosis univariante (K).

Se separó la muestra $(n=864)$ en dos partes en forma aleatoria: las respuestas de 461 participantes (53\%) se utilizaron para realizar un Análisis Factorial Exploratorio (AFE) mientras que sobre las respuestas de los 403 participantes restantes (47\%) Factorial Confirmatorio (AFC). De esta forma, la estructura hallada en el AFE se corroboró en el AFC. Se decidió un AFE dado que la 
muestra en la que se administró este instrumento detenta características diferentes a la descrita en el estudio original de Alarcón (2014) y por lo tanto, la dimensionalidad del instrumento podría, eventualmente, diferir.

El AFE se realizó con el programa Factor 10 (Ferrando \& Lorenzo Seva, 2017). Se verificó la pertinencia de este análisis mediante el coeficiente KaiserMeyer-Olkin (KMO) y la prueba de esfericidad de Bartlett. Dada la falta de normalidad multivariante de los datos, se implementó el método de mínimos cuadrados no ponderados robustos (Robust Unweighted Least Squares, RULS) para la extracción de los factores con base en la matriz de correlaciones policóricas (Ferrando \& Lorenzo-Seva, 2014). Se utilizó la implementación óptima del análisis paralelo (Timmerman \& Lorenzo Seva, 2011) para determinar el número de factores. El análisis paralelo ha mostrado consistentemente mayor precisión que otros métodos de extracción de factores (Gaskin \& Happell, 2014) e incluso robustez frente a desviaciones de la normalidad cuando las matrices son policóricas (Garrido, Abad \& Ponsoda, 2013).

Para evaluar cada modelo originado en cada eliminación progresiva de los ítems, se observó el índice de bondad de ajuste (Goodness of Fit Index, GFI) y la raíz media cuadrática residual (Root Mean Square Residual, RMSR). Se prestó especial atención a la magnitud de los residuos estandarizados (RE) eliminando los ítems con los mayores RE. Según el criterio de Hair, Anderson, Tatham y Black (1999), para que un modelo sea aceptable dicha magnitud debe ser menor que el valor absoluto de 2,58 y según el criterio de Kelley (1935), el RMSR debe rondar un valor medio esperado de $1 / \sqrt{n}$, en este caso $1 / \sqrt{461} \approx 0,047$. El cociente $1 / \sqrt{n}$ surge del error típico aproximado de un coeficiente de correlación nulo y que el RMSR sea cercano a dicho valor indicaría que no restan relaciones sistemáticas para continuar explorando (Ferrando \& Anguiano-Carrasco, 2010). Si bien en el modelo hallado se cumplió el criterio de Kelley, no fue así con el criterio de Hair et al. (1999), ya que el valor del RE fue de 2,60. Cumplir con el criterio de Hair et al. hubiera implicado la eliminación de dos ítems más, y se prefirió conservarlos, puesto que la diferencia con el cumplimiento de dicho criterio fue mínima.

Luego de explorar la estructura interna de la escala, se calculó el alfa ordinal, el índice de atenuación (Domínguez-Lara, 2017) y el índice alfa de Cronbach. Se verificó además que ningún reactivo, al quitarlo del conjunto definitivo de ítems, resulte en un aumento de los mencionados índices. 
Como se indicó previamente las respuestas de los 403 participantes restantes, seleccionados en forma aleatoria de la muestra, se utilizaron para realizar un AFC del modelo obtenido en el AFE. El AFC se implementó mediante el programa Mplus (Muthén \& Muthén, 2010) y al igual que en el AFE, el análisis se realizó con base en las matrices de correlaciones policóricas. Los parámetros fueron estimados con el método de mínimos cuadrados ponderados robustos (Weighted Least Squares Mean and Variance Adjusted, WLSMV) utilizando los siguientes índices de ajuste: índice de ajuste comparativo (Comparative Fit Index, CFI), índice de Tucker-Lewis (Tucker-Lewis Index, TLI) y la raíz media del error cuadrático de aproximación (Root Mean Square Error of Approximation, RMSEA), reseñados en la bibliografía como adecuados para datos ordinales (Abad, Olea, Ponsoda, \& García, 2011; Byrne, 2012). Se consideró el ajuste como satisfactorio si CFI y TLI $\geq 0,90$ y RMSEA $\leq 0,08$ (Abad, et al., 2011; Brown, 2015; Byrne, 2012). El límite superior del intervalo de confianza al 90\% del RMSEA tampoco debería exceder el valor 0,08 (Hu \& Bentler, 1998).

Por último, se exploró la relación de las variables sociodemográficas género, máximo nivel de educación alcanzado y zona de residencia dentro de Argentina con el puntaje de los sujetos en la versión argentina de la Escala de Gratitud de Alarcón (2014) mediante Análisis de la Varianza (ANOVA). Blanca, Alarcón, Arnau, Bono y Bendayan (2017) condujeron un estudio de simulación variando distintas condiciones, concluyendo que el estadístico $F$ del ANOVA es robusto en términos de error de Tipo I en todos los casos estudiados e independientemente de las condiciones manipuladas. El tamaño del efecto del ANOVA fue estimado mediante $\eta^{2}$ parcial, siendo sus criterios de interpretación (Cárdenas Castro \& Arancibia Martini, 2014): a) $\approx 0,01$ como pequeño, b) $\approx 0,06$ como mediano, c) $\approx 0,14$ como grande, Para el contraste post-hoc se utilizó la prueba de Scheffé donde el tamaño del efecto se valoró mediante el índice $d$ de Cohen, interpretando (Cohen, 1988): a) $\approx .20$ como pequeño, b) $0, \approx 0,50$ como moderado y c) $\approx 0,80$ como grande. Asimismo, se relevó la relación del puntaje de los participantes en la escala adaptada con la edad y con los puntajes de las dimensiones de la VAEFL y la ECP. En este caso se utilizó el coeficiente no paramétrico de Spearman (Badii, Guillen, Lugo, \& Garnica, 2014).

\subsection{Conflicto de interés}

Los autores manifiestan que no poseen conflictos de intereses. 


\section{RESULTADOS}

\subsection{Estadísticos Descriptivos}

Se pueden observar en la Tabla 1:

Tabla 1.

Estadísticos Descriptivos de los Ítems

\begin{tabular}{|c|c|c|c|c|}
\hline ÍTEMS & M & DE & AS & K \\
\hline 1.- Debemos agradecer el beneficio recibido por más pequeño que sea. & 4,4 & 0,6 & $-0,9$ & 0,6 \\
\hline 2.- Nunca olvidaré lo que otros han hecho por mí. & 4,5 & 0,5 & $-0,5$ & $-1,1$ \\
\hline 3.- Es una obligación moral agradecer lo que hemos recibido. & 4,2 & 0,8 & $-0,9$ & 0,9 \\
\hline 4.- No tengo porqué agradecer el favor que me han hecho. * & 4,4 & 0,6 & $-0,9$ & 0,6 \\
\hline 5.- La gratitud termina cuando se pagan los favores recibidos. ${ }^{*}$ & 4,0 & 0,9 & $-0,9$ & 0,8 \\
\hline 6.- Es difícil olvidar a quienes me hicieron un favor. & 4,2 & 0,8 & $-1,2$ & 2,4 \\
\hline 7.- Me es fácil elogiar a una persona bondadosa. & 4,0 & 0,8 & $-0,5$ & $-0,0$ \\
\hline 8.- Me considero una persona agradecida. & 4,1 & 0,7 & $-0,5$ & 0,2 \\
\hline $\begin{array}{l}\text { 9.- Me preocupo por corresponder a la persona que me hizo un favor cuando más } \\
\qquad \text { lo necesitaba. }\end{array}$ & 4,3 & 0,6 & $-0,2$ & $-0,6$ \\
\hline 10.- La recompensa a la persona que me ayudó pone fin a la gratitud que le debía. & 3,7 & 1,0 & $-0,8$ & 0,5 \\
\hline 11.- Me siento muy contento cuando retribuyo a la persona que me hizo un favor. & 4,3 & 0,6 & $-0,5$ & 0,1 \\
\hline 12.- Considero valiosos los beneficios que he recibido. & 4,2 & 0,6 & $-0,3$ & 0,4 \\
\hline 13.- Lo que somos y lo bueno que tenemos, no lo hemos conseguido solos. & 4,1 & 0,9 & $-1,0$ & 0,9 \\
\hline 14.- La persona generosa merece reconocimiento de los demás. & 3,9 & 0,9 & $-0,5$ & $-0,2$ \\
\hline 15.- La gratitud nunca termina. & 4,1 & 0,8 & $-0,6$ & $-0,1$ \\
\hline 16.- Guardo en la memoria a quienes generosamente me ayudaron. & 4,4 & 0,6 & $-0,5$ & 0,0 \\
\hline 17.- Es una obligación moral corresponder a quienes me ayudaron. & 3,9 & 0,9 & $-0,6$ & 0,4 \\
\hline 18.- Hay muchas cosas en la vida por las que estoy agradecido. & 4,5 & 0,6 & $-0,7$ & $-0,1$ \\
\hline
\end{tabular}

Nota. M: Media , DE: Desviación Estándar, As: índice de Asimetría, K: índice de Curtosis

*ítem redactado en sentido negativo

\subsection{Análisis Factorial Exploratorio}

Se realizó un primer AFE -pertinente dado que $\mathrm{KMO}=0,89$ y prueba de esfericidad de Bartlett: $\chi 2(153)=2526 ; p=0,00001-$ sobre los 18 ítems administrados. La implementación óptima del análisis paralelo indicó una estructura unifactorial y, a su vez, existió un único factor que explicó el $42 \%$ de la varianza. Por lo tanto, se impuso una estructura unifactorial, eliminándose los ítems con mayores residuos estandarizados en análisis consecutivos.

En la solución unifactorial alcanzada luego de la depuración el KMO fue de0,91 y la prueba de esfericidad de Bartlett tuvo los siguientes valores: $\chi 2$ $(66)=1652 ; p=0,00001$. Se conservaron 12 ítems, donde el mayor residuo estandarizado fue 2,60 y el RSMR tuvo un valor de0,056, que no difirió en gran medida del valor correspondiente al criterio de Kelley $(0,047)$. El valor de GFI fue de 0,99, señalando la adecuación del modelo explorado, siendo la 
varianza descripta por el mismo de 51,28\%. Se optó por conservar la escala con 12 ítems, ya que el cumplimiento estricto del criterio de Hair (residuos estandarizados menores a 2,58 ) y un RMSR mucho más aproximado a $1 / \sqrt{n}$ implicarían la eliminación de dos ítems más (ítems 1 y 13), resultando una prueba de 10 reactivos. Si se eliminaran dichos ítems, este modelo tendría un RMSR de 0,051 y un valor de mayor RE de 2,54. Considerando que se pierden dos reactivos de los 12 se optó por mantenerlos ya que se aproxima en forma muy cercana a los criterios planteados.

El alfa ordinal de la prueba fue de 0,91 con un índice de atenuación del 7\%, lo que indica que no hay diferencias importantes con el alfa de Cronbach (Domínguez-Lara, 2017). El alfa de Cronbach de la escala resultó de 0,85, indicando una consistencia interna muy buena según el criterio de DeVellis (2012). En todos los casos el alfa ordinal se mantuvo igual o disminuyó ante la eliminación del reactivo, como se observa en la Tabla 2, donde también se incluyen las cargas factoriales, así como el alfa de Cronbach y el índice de atenuación sin el ítem. Las cargas factoriales fueron, como mínimo, de 0,45. Se exponen los 12 ítems conservando su numeración original.

Tabla 2.

Cargas Factoriales y Alfa de Cronbach, Alfa Ordinal e Índice de Atenuación sin el Ítem

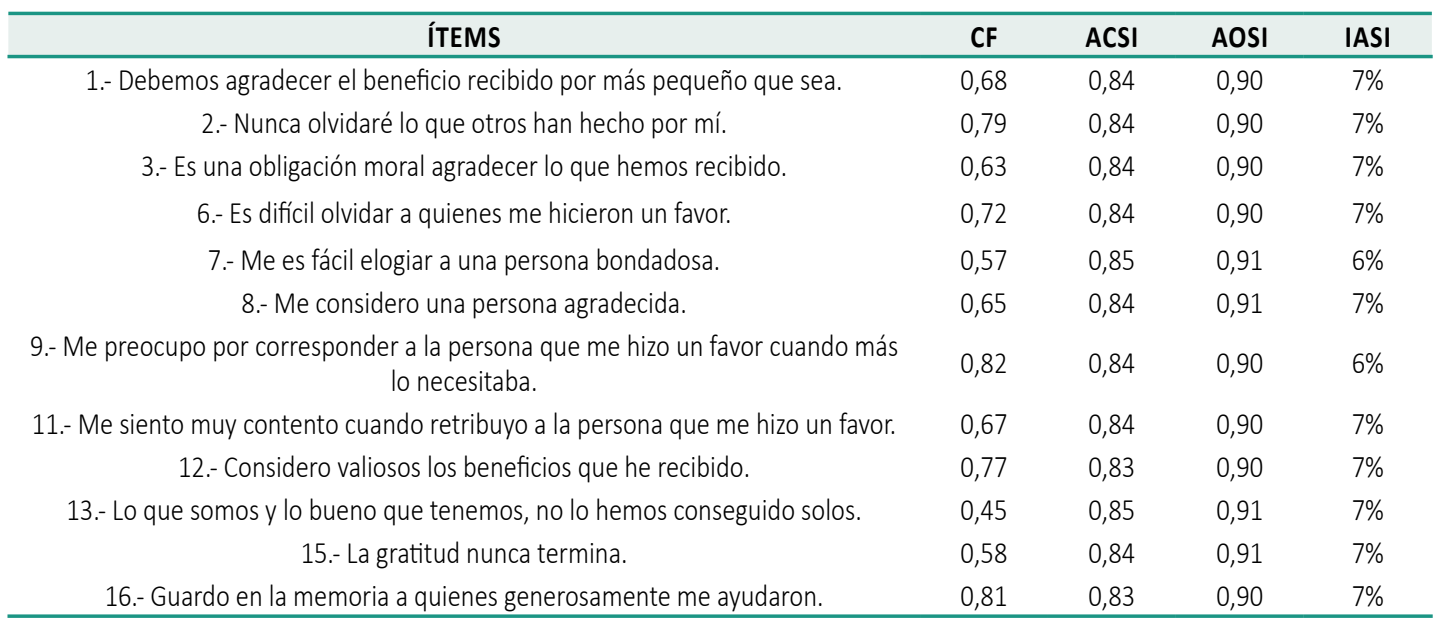

Nota. CF: Carga Factorial, ACSI: Alfa de Cronbach Sin el Ítem, AOSI: Alfa Ordinal Sin el Ítem. IASI: Índice de Atenuación Sin el Ítem.

\subsection{Análisis Factorial Confirmatorio}

Los índices de ajuste comparativos muestran un resultado muy adecuado para la verificación del modelo unidimensional ( $C F I=0,973 ; T L I=0,967)$. Si se considera el índice de ajuste absoluto RMSEA = 0,073 cumple con la condición de ser menor que 0,08 . Con respecto a su Intervalo de Confianza (IC) al 90\% 
resultó ser de 0,061-0,085). El límite superior del IC podría suponer cierto desajuste por no cumplir el criterio de ser menor a 0,08 (Hu \& Bentler, 1998). Sin embargo, es posible aceptar razonablemente la relativa unidimensionalidad del constructo para la versión argentina de la EG. Las estimaciones de los parámetros fueron estadísticamente significativas al $5 \%$ en todos los casos. Tal como se observa en la Figura 1, los pesos estandarizados de cada uno de los ítems fueron elevados.

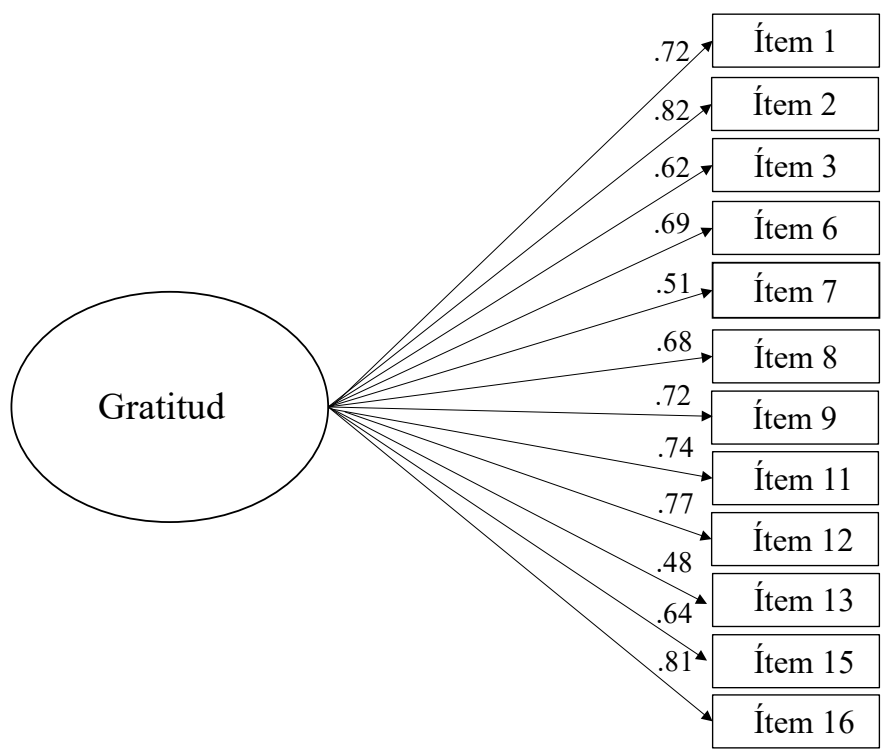

Figura 1. Modelo Factorial Confirmatorio de la Versión Argentina de la EG

\subsection{Relación con Variables Sociodemográficas}

Previamente a la realización de los ANOVA, se comprobó el supuesto de homocedasticidad mediante la prueba de Levene. El mismo se cumplió para género $(p=0,25)$, máximo nivel educativo alcanzado $(p=0,43)$ y zona de residencia $(p=0,45)$. Los puntajes de la EG, como se mencionó previamente, no tienden a distribuirse de forma normal multivariante.

En el ANOVA por género existieron diferencias significativas a favor de las mujeres $(p=0,008)$, sin embargo, el tamaño del efecto resultó muy pequeño ( $n 2$ parcial $=0,008$ ). Los ANOVA para máximo nivel educativo alcanzado y zona de residencia tuvieron un patrón similar de diferencias significativas con tamaños del efecto muy pequeños. En el primer caso con un valor $p$ de 0,007 y un $\eta 2$ parcial de 0,016 . En el ANOVA por zona de residencia el valor $p$ fue de 0,045 y el $\eta 2$ parcial de 0,011 . 
La prueba post-hoc de Scheffé indicó que existieron diferencias significativas, pero con tamaño del efecto muy pequeño a favor de quienes tienen un nivel universitario o técnico completo con respecto a quienes no lo han completado $(p=0,024, d$ de Cohen $=0,029)$. En cuando a zona de residencia la pequeña diferencia se dio entre residentes en el resto de las provincias argentinas y residentes en Gran Buenos Aires ( $p=0,082$, $d$ de Cohen $=0,025$ ).

La correlación de Spearman entre puntaje en Gratitud y edad resultó positiva y significativa, aunque de intensidad baja $(.17, p<0,0001)$.

\subsection{Validez Convergente: Relación de la Escala de Gratitud adaptada con la} Versión Argentina de la Escala de Felicidad de Lima y la Escala de Conducta Prosocial

Las correlaciones de Spearman entre dimensiones de la EG y dimensiones de la VAEFL y la ECP fueron todas positivas y de magnitud pequeña, pero estadísticamente significativa. La correlación más alta fue con la dimensión Confortar de la ECP, como se expone en la Tabla 3.

Tabla 3.

Análisis de Validez Convergente

\begin{tabular}{cccccc}
\hline & VAEFL & \multicolumn{2}{c}{ ECP } \\
\hline SVPE & SVPI & SCV & AY & CO \\
EG & $0,21^{* *}$ & $0,24^{* *}$ & $0,29^{* *}$ & $0,25^{* *}$ & $0,38^{* *}$ \\
\hline
\end{tabular}

Nota. EG: Escala de Gratitud, VAEFL: Versión Argentina de la Escala de Felicidad de Lima, SVPE: Dimensión de la VAEFL Sentido de la Vida Perspectiva Externa, SVPI: Dimensión de la VAEFL Sentido de la Vida Perspectiva Interna, SCV: Dimensión de la VAEFL Satisfacción Con la Vida, ECP: Escala de Conducta Prosocial, AY: Dimensión de la ECP Ayuda, CO: Dimensión de la ECP Confortar.

$\left[{ }^{* *}\right] p<0,01$

\section{DISCUSIÓN}

El presente estudio tuvo como objetivo la adaptación a la Argentina de la Escala de Gratitud por el psicólogo peruano Reynaldo Alarcón en 2014. Este es el primer instrumento para medir la Gratitud construido en un país latinoamericano. Aunque ha sido utilizado para realizar estudios psicológicos empíricos en Perú (e.g. Alarcón \& Caycho, 2015; León \& Hidalgo, 2017; Menautt Champi, Checa Cano, \& Arias Gallegos, 2016), aún no se conocen adaptaciones a otros países.

En la adaptación argentina de la EG, la estructura factorial del instrumento original se vio modificada: mientras que la estructura original era trifactorial, los puntajes de la escala adaptada brindaron evidencias de unidimensionalidad. Una posible razón de este cambio es que la cantidad de factores puede 
variar entre diversas culturas y subpoblaciones, teniendo la gratitud en la muestra argentina una estructura psicométrica más simple que en la muestra peruana recolectada por Alarcón (2014). Como se ha mencionado en la introducción, no existe consenso acerca de la dimensionalidad de la gratitud. En el estudio de McCullough et al. (2002) se halla una estructura unifactorial para la gratitud y en el mismo artículo original de Alarcón (2014) el primero de los tres factores encontrados explica más del $40 \%$ de la varianza. Además, uno de los factores de la escala original tiene solo dos ítems, por lo cual es, al menos, discutible considerarlo como tal (Lloret-Segura, Ferreres-Traver, Hernández-Baeza \& Tomás-Marco, 2014).

Es necesario destacar que hubo diferencias metodológicas en el método de extracción de factores entre el análisis factorial de la escala original y el de la adaptación argentina, ya que las recomendaciones al respecto han cambiado en los últimos años (Ferrando \& Lorenzo-Seva, 2014; Izquierdo, Olea, \& Abad, 2014). El Análisis de Componentes Principales, utilizado por Alarcón (2014), tiende a sobreestimar el número de factores. Esto puede haber influido en las diferencias encontradas. Lo cierto es que la estructura unifactorial en la muestra argentina ha resultado no solo de una exploración de los datos, sino que además se ha corroborado mediante AFC, mostrando adecuados índices de ajuste.

Los ítems eliminados correspondieron mayoritariamente (tres de los seis ítems descartados) al factor Obligación Moral de la escala original. Se podría inferir que el componente moral de la gratitud no ocupa un lugar tan preponderante como en el modelo planteado por Alarcón. Aun así, aparece reflejado por algunos de los ítems que fueron sostenidos tras el proceso de depuración. Igualmente, las diferencias en el procedimiento de depuración aplicado hacen difícil la comparación. Dos de los ítems eliminados son los que componían el factor Cualidad Sentimental en la escala original que, como ya se mencionó, es cuestionable en su calidad de dimensión.

En cuanto a las evidencias de validez convergente, la escala tuvo la correlación más alta con la dimensión Confortar de la ECP, vinculada a sentimientos y acciones empáticas. También tuvo correlaciones más bajas, aunque positivas y significativas, con la dimensión Ayuda de la ECP y con todas las dimensiones de la VAEFL. Estos resultados son coherentes con hallazgos previos, según los cuales la gratitud se asocia con el comportamiento prosocial y la felicidad (e.g. Alarcón \& Caycho, 2015; Ma, Tunney, \& Ferguson, 2017; Safaria, Diponegoro, \& Bashori, 2017; Yost-Dubrow \& Dunham, 2017). 
Dado que las opciones de la escala Likert de respuesta son ordinales, se calculó el alfa ordinal, pero asimismo se calculó el alfa de Cronbach por haberlo utilizado Alarcón y para poder comparar con el mismo índice. Posteriormente se comprobó que el alfa de Cronbach no está distorsionado por ser el índice de atenuación de $7 \%$ o menor, es decir, muy inferior que el límite de 30\% (Domínguez Lara, 2017). Lo que significa que ambos índices son muy aproximados. Además, los valores de cada uno de ellos señalan que la consistencia interna de la escala es muy buena.

Las relaciones de la gratitud con las variables sociodemográficas -género, máximo nivel educativo alcanzado y zona de residencia-fueron significativas, pero tuvieron tamaños del efecto muy pequeños. En consecuencia, pueden interpretarse como espurias y causadas por el elevado tamaño muestral. La falta de relación entre género y gratitud es coherente con los hallazgos de Alarcón y Caycho (2015), que aplicaron las versiones originales de los mismos instrumentos administrados en el presente estudio. Otras investigaciones sobre el tema tampoco han encontrado relación significativa entre género y gratitud (e.g. Feng, 2011, en pastores norteamericanos de ascendencia coreana y sus esposas) y otros, diferencias a favor de las mujeres (e.g. Froh, Yurkewicz, \& Kashdan, 2009, en estudiantes norteamericanos de nivel secundario). En cuanto a la falta de relación entre la gratitud y el nivel educativo podría deberse a que, aunque la primera puede ser aprendida, en general lo es en la educación informal, la cual se realiza en el seno de la familia ( $L$ i, 2015). No existen evidencias previas que hagan esperar diferencias significativas en gratitud en relación a la zona de residencia. Iguales consideraciones pueden realizarse con respecto a la relación entre edad y gratitud.

En suma, la versión argentina de la EG ha mostrado propiedades psicométricas aceptables. Dada la relación negativa de la gratitud con el neuroticismo, la depresión y la tasa de suicidio, esta escala puede ser utilizada en intervenciones de corte clínico y psicosocial. Al ser unifactorial, el puntaje total de la escala es el puntaje en gratitud, siendo así muy sencilla para administrar y puntuar.

La limitación de esta investigación es principalmente el carácter no probabilístico del muestreo, por lo cual se condiciona la generalización de los resultados. Ante la dificultad para recolectar muestras de carácter probabilístico, es posible, en futuras investigaciones, administrar la versión adaptada de la EG a grupos puntuales donde sea esperable un nivel de gratitud especialmente elevado o bajo. De esta manera se obtendrían evidencias de validez 
relacionada con un criterio externo. Además, se continuará este estudio con la exploración de la existencia de funcionamiento diferencial del ítem según género y la modelización de la escala mediante modelos de la Teoría de la Respuesta al Ítem para ítems politómicos, específicamente el Modelo de Respuesta Graduada y el Modelo de Crédito Parcial.

Financiamiento: Esta investigación fue financiada por los subsidios de la Universidad de Buenos Aires UBACyT 2018 Código № $20020170100200 B$ B y UBACyT Código № 20020170200001BA y de la Agencia Nacional de Promoción Científica y Tecnológica PICT-2017-3226.

Agradecimientos: A la Universidad de Buenos Aires por el otorgamiento de los Subsidios UBACYT, a la Agencia Nacional de Promoción Científica y Tecnológica por el otorgamiento del Subsidio PICT y al Consejo Nacional de Investigaciones Científicas y Técnicas (CONICET) por la beca posdoctoral adjudicada a la primera autora.

\section{REFERENCIAS}

Abad, F., Olea, J., Ponsoda, V., \& García, C. (2011). Medición en ciencias sociales y de la salud. Madrid, España: Síntesis. URL: https://dialnet.unirioja.es/servlet/libro?codigo $=552272$

Adler, M. G., \& Fagley, N. S. (2005). Appreciation: Individual differences in finding value and meaning as a unique predictor of subjective well-being. Journal of Personality, 73(1), 79-114. https://doi.org/10.1111/j.1467-6494.2004.00305.x

Alarcón, R. (2006). Desarrollo de una escala factorial para medir la felicidad. Revista Interamericana de Psicología, 40(1), 95-102. URL: http://pepsic.bvsalud.org/ scielo.php?script=sci_arttext\&pid=S0034-96902006000100010\&Ing=pt\&nr$\mathrm{m}=$ iso

Alarcón, R. (2014). Construcción y valores psicométricos de una escala para medir la gratitud. Acta de Investigación Psicológica, 4(2), 1520-1534. https://doi. org/10.1016/S2007-4719(14)70391-6

Alarcón, R. \& Caycho, T. (2015). Relaciones entre gratitud y felicidad en estudiantes universitarios de Lima Metropolitana. Psychologia: Avances de la Disciplina, 9(1), 59-69. http://dx.doi.org/10.21500/19002386.993

Ato, M., López, J. J., \& Benavente, A. (2013). Un sistema de clasificación de los diseños de investigación en psicología. Anales de Psicología, 29(3), 1038-1059. http:// dx.doi.org/10.6018/analesps.29.3.178511

Auné, S. E., Abal, F. J. P., \& Attorresi, H. F. (2016). Diseño y construcción de una escala de conducta prosocial para adultos. Revista Iberoamericana de Diagnóstico y Evaluación Psicológica, 42(2), 15-25. http://dx.doi.org/10.21865/RIDEP42_15

Auné, S. E., Abal, F. J. P., \& Attorresi, H. F. (2017). Versión argentina de la Escala de Felicidad de Lima. Diversitas: Perspect. Psicol., 13(2), 201-214. http://dx.doi. org/10.15332/s1794-9998.2017.0002.05

Auné, S. E. \& Attorresi, H. F. (2017). Dimensionalidad de un test de conducta prosocial. Evaluar, 17(1), 29-37. URL: https://revistas.unc.edu.ar/index.php/revaluar/ article/view/17072 
Auné, S. E., Blum, G. D., Abal, F. J. P., Lozzia, G., \& Attorresi, H. F. (2014). La conducta prosocial: Estado actual de la investigación. Perspectivas en Psicología, 11(2), 21-33. URL: http://www.seadpsi.com.ar/revistas/index.php/pep/article/view/153

Badii, A., Guillen, O. P., Lugo, S., \& Garnica, J. A. (2014). Correlación No-Paramétrica y su Aplicación en las Investigaciones Científica. International Journal of Good Conscience, 9(2), 31-40. URL: http://www.spentamexico.org/v9-n2/A5.9(2)31-40. pdf

Bartlett, M. Y., Condon, P., Cruz, J., Baumann, J., \& Desteno, D. (2012). Gratitude: Prompting behaviours that build relationships. Cognition \& Emotion, 26(1), 2-13. https://doi.org/10.1080/02699931.2011.561297

Bernabé-Valero, G., García-Alandete, J., \& Gallego-Pérez, J. F. (2014). Construcción de un cuestionario para la evaluación de la gratitud: El cuestionario de Gratitud-20 ítems (G-20). Anales de Psicología, 30(1), 278-286. http://dx.doi.org/10.6018/ analesps.30.1.135511

Blanca, M. J., Alarcón, R., Arnau, J., Bono, R., \& Bendayan, R. (2017). Non-normal data: Is ANOVA still a valid option? Psicothema, 29(4), 552-557. http://dx.doi. org/10.7334/psicothema2016.383

Brown, T. (2015). Confirmatory factor analysis for applied research (2nd Ed.). Nueva York, Estados Unidos: Guilford Press. URL: https://www.guilford. com/books/Confirmatory-Factor-Analysis-for-Applied-Research/TimothyBrown/9781462515363

Byrne, B. M. (2012). Structural equation modeling with Mplus: Basics, concepts, applications, and programming. Nueva York, Estados Unidos: Routledge. URL: https://www.amazon.es/Structural-Equation-Modeling-Mplus-Applications/ $\mathrm{dp} / 1848728395$

Cárdenas Castro, M., \& Arancibia Martini, H. (2014). Potencia estadística y cálculo del tamaño del efecto en $\mathrm{G}^{*}$ Power: complementos a las pruebas de significación estadística y su aplicación en psicología. Salud \& Sociedad, 5(2), 210-244. http:// doi.org/10.22199/S07187475.2014.0002.00006

Cohen, J. (1988). Statistical Power Analysis for the Behavioral Sciences. 2nd Edit., Hillsdale, N.J., Erlbaum. Nueva York, Estados Unidos: Academic Press. URL: http:// www.utstat.toronto.edu/ brunner/oldclass/378f16/readings/CohenPower.pdf

DeCarlo, L. T. (1997). On the meaning and use of kurtosis. Psychological Methods, 2(3), 292-307. http://dx.doi.org/10.1037/1082-989X.2.3.292

DeVellis, R. (2012). Scale development. Theory and applications. Nueva York, Estados Unidos: SAGE. URL: https://www.amazon.es/Scale-Development-ApplicationsApplied-Research/dp/1412980445

Diener, E., Emmons, R. A., Larsen, R. J., \& Griffin, S. (1985). The Satisfaction with Life Scale. Journal of Personality Assessment, 49(1), 71-75. https://doi.org/10.1207/ s15327752jpa4901_13

Digman, J. M. (1990). Personality structure: Emergence of the five-factor model. Annual Review of Psychology, 41(1), 417-440. https://doi.org/10.1146/annurev. ps.41.020190.002221

Disabato, D. J., Kashdan, T. B., Short, J. L., \& Jarden, A. (2016). What predicts positive life events that influence the course of depression? A longitudinal examination of gratitude and meaning in life. Cognitive Therapy and Research. Advance online publication. http://dx.doi.org/10.1007/s10608-016-9785-x

Domínguez-Lara, S. (2017, en prensa). Fiabilidad y alfa ordinal. Actas Urológicas Españolas. http://dx.doi.org/10.1016/j.acuro.2017.07.002 
Emmons, R. A., \& McCullough, M. E. (2003). Counting blessings versus burdens: an experimental investigation of gratitude and subjective well-being in daily life. Journal of Personality and Social Psychology, 84(2), 377-389. http://dx.doi. org/10.1037/0022-3514.84.2.377

Emmons, R. A., \& Mishra, A. (2011). Why gratitude enhances well- being: What we know, what we need to know. In M. Kennon, T. Sheldon, T. Kashdan, \& M. F. Steger (Eds), Designing positive psychology: Taking stock and moving forward (pp.248264). Nueva York, Estados Unidos: Oxford Press. http://dx.doi.org/10.1093/ acprof:oso/9780195373585.003.0016

Feng, E. (2011). The impact of spiritual well-being, gratitude, and loneliness on marital satisfaction among Korean American pastors and spouses. (M. S. Thesis). California State University, Long Beach. URL: https://search.proquest.com/openview/6f5e5d9edce707b23cc55baa8a5adecc/1?pq-origsite=gscholar\&cbl=18750\&diss=y

Ferrando, P. J., \& Anguiano-Carrasco, C. (2010). El análisis factorial como técnica de investigación en psicología. Papeles del Psicólogo, 31(1), 18-33. URL: http://www. redalyc.org/articulo.oa?id=77812441003

Ferrando, P. J., \& Lorenzo-Seva, U. (2014). El análisis factorial exploratorio de los ítems: algunas consideraciones adicionales. Anales de Psicología, 30(3), 1170-1175. http://dx.doi.org/10.6018/analesps.30.3.199991

Ferrando, P. J., \& Lorenzo-Seva, U. (2017). Program FACTOR at 10: Origins, development and future directions. Psicothema, 29(2), 236-240. http://dx.doi. org/10.7334/psicothema2016.304

Froh, J., Yurkewicz, Ch., \& Kashdan, T. (2009). Gratitude and subjective well-being in early adolescence. Examining gender differences. Journal of Adolescence, 32(3), 633-650. http://dx.doi.org/10.1016/j.adolescence.2008.06.006

Garrido, L. E., Abad, F. J., \& Ponsoda, V. (2013). A new look at Horn's parallel analysis with ordinal variables. Psychological Methods, 18(4), 454-474. http://dx.doi. org/10.1037/a0030005

Gaskin, C. J., \& Happell, B. (2014). On exploratory factor analysis: A review of recent evidence, an assessment of current practice, and recommendations for future use. International Journal of Nursing Studies, 51(3), 511-521. http://dx.doi. org/10.1016/j.ijnurstu.2013.10.005

Gil-Escudero, G., \& Martínez-Arias, M. R. (2001). Metodología de encuestas. En M. J. Navas (Ed.), Métodos, diseños y técnicas de investigación psicológica ( $\mathrm{pp}$. 379-436). Madrid, España: Universidad Nacional de Educación a Distancia. URL: http://www.sidalc.net/cgi-bin/wxis.exe/?IsisScript=UCC.xis\&method=post\&formato $=2 \&$ cantidad $=1$ \& expresion $=\mathrm{mfn}=135536$

Gulliford, L., Morgan, B., \& Kristjánsson, K. (2013). Recent work on the concept of gratitude in philosophy and psychology. Journal of Value Inquiry, 47(3), 285-317. http://dx.doi.org/10.1007/s10790-013-9387-8

Hair, J. F., Anderson, R.E., Tatham, R. L. \& Black, W. C. (1999). Análisis Multivariante. Madrid, España: Prentice Hall Iberia. URL: https://wwwyyy.files.wordpress. com/2018/01/anc3a1lisis-multivariante-de-joseph-f-hair-y-otros.pdf

Hammer, J. H., \& Brenner, R. E. (2017). Disentangling Gratitude: A Theoretical and Psychometric Examination of the Gratitude Resentment and Appreciation TestRevised Short (GRAT-RS). Journal of Personality Assessment, 1-10. http://dx.doi. org/10.1080/00223891.2017.1344986

Hu, L., \& Bentler, P. M. (1998). Fit indices in covariance structure modeling: sensitivity to under parameterized model misspecification. Psychological Methods, 3(4), 424-453. URL: http://psycnet.apa.org/buy/1998-11538-003 
Israel-Cohen, Y., Uzefovsky, F., Kashy-Rosenbaum, G., \& Kaplan, O. (2015). Gratitude and PTSD symptoms among Israeli youth exposed to missile attacks: examining the mediation of positive and negative affect and life satisfaction. The Journal of Positive Psychology, 10(2), 99-106. http://dx.doi.org/10.1080/17439760.2014.9 2791

Izquierdo, I., Olea, J., \& Abad, F. J. (2014). Exploratory factor analysis in validation studies: Uses and recommendations. Psicothema, 26(3), 395-400. http://dx.doi. org/10.7334/psicothema2013.349

Kelley, T. L. (1935). Essential traits of mental life. Cambridge, MA: Harvard University Press.

Kleiman, E. M., Adams, L. M., Kashdan, T. B., \& Riskind, J. H. (2013). Gratitude and grit indirectly reduce risk of suicidal ideations by enhancing meaning in life: Evidence for a mediated moderation model. Journal of Research in Personality, 47(5), 539-546. http://dx.doi.org/10.1016/j.jrp.2013.04.007

Joseph, S., \& Wood, A. M. (2010). Assessment of positive functioning in clinical psychology: Theoretical and practical issues. Clinical Psychology Review, 30(7), 830-838. http://dx.doi.org/10.1016/j.cpr.2010.01.002

Lambert, N. M., Fincham, F. D. \& Stillman, T. F. (2012). Gratitude and depressive symptoms: The role of positive reframing and positive emotion. Cogn. Emot. 26(4), 615-633. http://dx.doi.org/10.1080/02699931.2011.595393

León, S. \& Hidalgo, E. (2017). Gratitud y felicidad en estudiantes del segundo al quinto año de una universidad privada de Lima este (Tesis para licenciatura). Universidad Peruana Unión, Lima. URL: http://repositorio.upeu.edu.pe/handle/UPEU/424

Li, S. (2015). Chinese parents' role modeling: promoting gratitude. Childhood Education, 91(3), 190-197. http://dx.doi.org/10.1080/00094056.2015.1047310

Li, D., Zhang, W., Li, X., Li, N., \& Ye, B. (2012). Gratitude and suicidal ideation and suicide attempts among Chinese Adolescents: Direct, mediated, and moderated effects. Journal of Adolescence, 35, 55-66. http://dx.doi.org/10.1016/j.adolescence.2011.06.005

Lloret-Segura, S., Ferreres-Traver, A., Hernández-Baeza, A., \& Tomás-Marco, I. (2014). El análisis factorial exploratorio de los ítems: una guía práctica, revisada y actualizada. Anales de Psicología, 30(3), 1151-1169. http://dx.doi.org/10.6018/ analesps.30.3.199361

Ma, L. K., Tunney, R. J., \& Ferguson, E. (2017). Does gratitude enhance prosociality? A meta-analytic review. Psychological Bulletin, 143(6), 601-635. http://dx.doi. org/10.1037/bul0000103

Mardia, K. V. (1970). Measures of multivariate skewnees and kurtosis with applications. Biometrika, 57(3), 519-530. http://dx.doi.org/10.1093/biomet/57.3.519

McCullough, M. E., Emmons, R. A., \& Tsang, J. A. (2002). The grateful disposition: a conceptual and empirical topography. Journal of Personality and Social Psychology, 82(1), 112-127. http://dx.doi.org/10.1037/0022-3514.82.1.112

Menautt Champi, C. F., Checa Cano, S. A., \& Arias Gallegos, W. L. (2016). La gratitud y la percepción de equidad en el trabajo como predictores de la motivación laboral en una agencia bancaria de Arequipa. Rev. Psicol. Arequipa. Univ. Catól. San Pablo, 6(1), 67-90. URL: http://ucsp.edu.pe/investigacion/psicologia/wp-content/ uploads $/ 2017 / 04 /$ Rev-psicol-UCSP-2016-1.pdf\#page $=67$

Montero, I., \& León, O. G. (2007). Sistema de clasificación del método en los informes de investigación en Psicología. International Journal of Clinical and Health Psychology, 5(1), 115-127. URL: http://www.redalyc.org/articulo.oa?id=33701007 
Muthén, L. \& Muthén, B. (2010). Mplus User's Guide, 6th Edn. Los Angeles, CA: Muthén \& Muthén. URL: https://www.statmodel.com/download/usersguide/ Mplus\%20user\%20guide\%20Ver_7_r6_web.pdf

Safaria, T., Diponegoro, A. M., \& Bashori, K. (2017). The Efficacy of Gratitude and Forgiveness Training to Increase Happiness. The Social Sciences, 12(11), 1948-1951. URL: http://docsdrive.com/pdfs/medwelljournals/sscience/2017/1948-1951.pdf

Tabachnick, B. \& Fidell, L. (2001). Using multivariate statistics. Fourth edition. Boston: Allyn and Bacon. URL: https://trove.nla.gov.au/work/7185329

Thomas, M., \& Watkins, P. (2003). Measuring the grateful trait: Development of the revised GRAT. In Poster session presented at the annual convention of the western psychological association, Vancouver, BC. http://dx.doi.org/10.13072/midss.100

Timmerman, M. E., \& Lorenzo-Seva, U. (2011). Dimensionality assessment of ordered polytomous items with parallel analysis. Psychological Methods, 16, 209-220. http://dx.doi.org/10.1037/a0023353

Van Dusen, J. P., Tiamiyu, M. F., Kashdan, T. B., \& Elhai, J. D. (2015). Gratitude, depression and PTSD: Assessment of structural relationships. Psychiatry Research, 230, 867-870. http://dx.doi.org/10.1016/j.psychres.2015.11.036

Watkins, P. C., Woodward, K., Stone, T., \& Kolts, R. L. (2003). Gratitude and happiness: development of a measure of gratitude, and relationships with subjective well-being. Social Behavior and Personality: an International Journal, 31(21), 431-451. http://dx.doi.org/10.2224/sbp.2003.31.5.431

Watson, D., Clark, L. A., \& Tellegen, A. (1988). Development and validation of brief measures of positive and negative affect: The PANAS scales. Journal of Personality and Social Psychology, 54(6), 1063-1070. URL: http://psycnet.apa.org/buy/198831508-001

Wood, A. M., Froh, J. J., \& Geraghty, A. W. A. (2010). Gratitude and well-being: A review and theoretical integration. Clinical Psychology Review, 30(7), 890-905. http://dx.doi.org/10.1016/j.cpr.2010.03.005

Wood, A. M., Maltby, J., Gillett, R., Linley, P. A., \& Joseph, S. (2008). The role of gratitude in the development of social support, stress, and depression: Two longitudinal studies. Journal of Research in Personality, 42(4), 854-871. https://doi. org/10.1016/j.jrp.2007.11.003

Yost-Dubrow, R., \& Dunham, Y. (2017). Evidence for a relationship between trait gratitude and prosocial behaviour. Cognition and Emotion, 1-7. http://dx.doi. org/10.1080/02699931.2017.1289153 